\title{
Efectos de la crisis económica en el empleo de la población inmigrante en el País Vasco: un análisis por sexo, formación y origen
}

\author{
Vicente Núñez-Antón, Ainhoa Oguiza-Tovar \\ y Jorge Virto-Moreno
}

\author{
Universidad del País Vasco, España
}

\section{Resumen}

En este artículo se ha realizado un estudio sobre las tasas de ocupación de la población inmigrante en el País Vasco en un periodo de expansión económica y otro de recesión. El objetivo ha consistido en analizar el comportamiento de la ocupación de la población inmigrante durante la crisis. Independientemente de su sexo, origen o cualificación, al final de la crisis la tasa de ocupación de los inmigrantes es menor que la de la población vasca, con la única excepción de las mujeres de procedencia americana. Las mujeres inmigrantes se han visto menos afectadas por la crisis que los hombres siendo la segregación sectorial la causa que ha podido tener un mayor peso a la hora de explicar la caída en la brecha de género en la ocupación. Con respecto al país de origen y el nivel de estudios son los trabajadores africanos y los de estudios primarios, foráneos y autóctonos, los grandes damnificados por la crisis.

Palabras clave: Inmigración, tasa de ocupación, nivel de estudios, género, crisis económica.

\section{Abstract}

Effects of the economic crisis on employment among the immigrant population in the Basque Country: an analysis for sex, qualification and geographical origin

In this paper we study employment rates among the immigrant population in the Basque Country in a period of economic expansion and in a recession. The objective is to analyze how employment among the immigrant population behaved during the crisis. Regardless of their gender, origin or qualifications, at the end of the crisis the employment rate among immigrants was lower than among the local-born Basque population, with the sole exception of women from the Americas. Immigrant women were less affected by the crisis than men. Sectoral segregation is probably the main cause of the fall in the gender gap in employment. With regard to country of origin and level of education, African workers and those with only basic studies (foreign and local-born alike) were hit hardest by the crisis.

Key words: Immigration, employment rate, educational level, gender, economic crisis. 


\section{INTRODUCCIÓN}

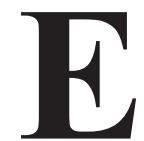

1 País Vasco es una región española situada en el extremo nororiental de la franja cantábrica, lindando al norte con el mar Cantábrico y Francia, y al sur, este y oeste con diversas regiones españolas. Si bien el País Vasco no ha sido un receptor tradicional de inmigrantes, de forma análoga al resto de España, se ha convertido en un destino importante de población extranjera en los últimos años, con menor intensidad y algo de retraso respecto a otras regiones españolas. Esta elevada entrada de inmigrantes ha tenido importantes efectos económicos y demográficos. El Observatorio Vasco de inmigración del Gobierno Vasco (Ikuspegi, 2015), basándose en datos y cifras oficiales, analiza el impacto de la inmigración extranjera en el País Vasco, por un lado, desde el punto de vista económico y, por otro, desde el punto de vista demográfico. En relación con el impacto económico, una vez observadas las cuentas públicas el estudio concluye que, tanto en 2008 como en 2012, los ingresos que genera la población de nacionalidad extranjera a las arcas públicas es superior al gasto que este colectivo le supone al sistema de bienestar, todo ello, a pesar de la crisis económica. Con respecto al impacto demográfico concluye que el mismo ha sido positivo en todas sus dimensiones. La inmigración extranjera ha permitido detener la pérdida de población que el País Vasco sufre desde la década de 1980, compensando la pérdida de población autóctona y permitiendo un crecimiento de la población. En el año 2005, el porcentaje de población extranjera en el País Vasco era de 3.4 por ciento, en 2008 de 5.4 por ciento y en 2012 de 6.3 por ciento, casi duplicándose dicho porcentaje entre 2005 y 2012. En resumen, se observa que el crecimiento natural de la población del País Vasco se ha producido gracias al aporte demográfico de la población extranjera.

En el año 2008 la economía vasca, al igual que la española y otras muchas economías mundiales, después de un largo periodo de expansión económica comenzó un periodo de recesión, con importantes caídas de la producción y destrucción del empleo, afectando de manera acusada las tasas de ocupación de la población. El Producto Interior Bruto (PIB) del País Vasco en el año 2005 era de 55943878 millones de euros, en el año 2008 era de 66178518 millones de euros y en 2012, 64262023 millones de euros. En el presente trabajo, estudiamos el periodo 2005-2008, que comprende una época de expansión económica y la posterior entrada en la 
recesión económica, periodo 2009-2012. El propósito de este artículo es evaluar cómo la recesión económica ha afectado a la población inmigrante en el País Vasco, con un enfoque especial en los cambios en las tasas de empleo de los no nacionales durante la crisis económica, diferenciando los dos periodos citados (2005-2008 y 2009-2012). La ocupación es un factor crítico para el bienestar de las familias inmigrantes, en especial en tiempos de crisis, cuando las ayudas públicas disminuyen y el trabajo es la principal, sino única, fuente de ingresos. Además, las caídas en la ocupación durante los periodos de crisis suelen ser más adversas en los segmentos de población más vulnerables como los inmigrantes o las mujeres. De esta forma, estamos interesados en evaluar hasta qué punto la recesión puede haber perjudicado aún más a los inmigrantes en el mercado laboral, con respecto a la población autóctona, examinando el impacto diferencial según nivel de estudios y región de origen para hombres y mujeres.

El artículo se estructura de la siguiente manera: la siguiente sección revisa los estudios existentes que investigan los vínculos entre empleo, sexo y nivel de educación sobre la ocupación en tiempos de crisis. A continuación se describen los datos utilizados en el análisis. Posteriormente, se analiza la evolución de la población inmigrante en el País Vasco y sus tasas de ocupación según las distintas características de los trabajadores analizados. La sección final ofrece algunas conclusiones.

\section{MARCo TEÓRICO Y EMPíRICO}

Los análisis de las crisis pasadas y de la gran recesión actual normalmente atribuyen a tres posibles explicaciones la diferente vulnerabilidad en el mercado laboral de los distintos grupos de individuos frente a las recesiones económicas: i) las diferencias en la exposición inicial de los trabajadores al shock, ii) las decisiones de empleo de las empresas durante una recesión, y iii) el comportamiento laboral de los trabajadores en respuesta a la disminución del ingreso familiar (Cho y Newhouse, 2013).

\section{Diferencias en la exposición al shock}

Las crisis económicas suelen afectar con más fuerza a los colectivos más vulnerables de la población como jovenes, inmigrantes o mujeres. La reciente recesión económica tiene una característica saliente ya que, a diferencia de crisis anteriores, los hombres han resultado más perjudicados en términos de empleo, probablemente debido a los sectores económicos en los que se emplean. 
La pérdida de empleo ha sido mucho mayor en los hombres, con mayor presencia en los sectores más afectados por la crisis (industria y construcción) que en las mujeres, más concentradas en el sector servicios, menos afectado por la crisis. Este patrón es común en los mercados de trabajo europeos, véase por ejemplo, los distintos artículos recopilados en Eydoux et al. (2014), incluido un análisis del mercado laboral español, similar al del País Vasco (Peña-Boquete, 2014). Este resultado sigue manteniendose incluso si nos restringimos a la población inmigrante (Farris 2015). Un alto nivel educativo ha podido ser también una especie de escudo anti-crisis. Los inmigrantes con mayor nivel de estudios parecen estar más protegidos en esta crisis (para una revisisón de la evidencia empírica internacional véase, por ejemplo, Psacharopoulos y Patrinos, 2002). Por último, diferentes grupos étnicos de inmigrantes pueden encontrarse con diferentes ventajas/desventajas según su similitud cultural con la población autóctona (hipótesis de asimilación segmentada, Portes y Zhou, 1993). Por tanto, el origen de procedencia de los inmigrantes puede ser un factor importante a la hora de explicar los distintos resultados obtenidos en el mercado laboral.

\section{Decisiones de empleo de las empresas}

La percepción de las empresas de la productividad de los distintos empleados puede afectar al mantenimiento del puesto de trabajo. Trabajadores de un nivel educativo bajo o medio pueden ser vistos como poco productivos y aumentar su probabilidad de despido. Los inmigrantes también pueden estar más desfavorecidos en el proceso de contratación durante una crisis. Por ejemplo, cuando hay una gran cantidad de solicitantes de empleo el dominio del idioma del país de acogida puede ser utilizado más extensamente como criterio de selección por parte de la empresa (Chaloff et al., 2012). Las distintas regulaciones laborales también pueden ser un factor importante. Los inmigrantes, por su reciente incorporación al mercado laboral, suelen tener menor seguridad contractual y a menudo tienen contratos temporales (en España al comienzo de la crisis casi la mitad de los inmigrantes tenían contratos temporales).

\section{Respuesta laboral de los trabajadores}

Dentro de esta categoría estaría el efecto trabajador añadido, el aumento de la participación laboral femenina para compensar la pérdida de renta familiar. Por esta razón la participación femenina en el mercado laboral suele resultar contra-cíclica (Sabarwal et al., 2011). 
Como se ha visto, estos tres factores vinculan el sexo, la educación, el origen y el sector económico en el que trabajan los inmigrantes con la vulnerabilidad ante la crisis. A lo largo de este estudio vamos a analizar las distintas tasas de ocupación de la población inmigrante con respecto a estas características.

\section{Datos}

El análisis que proponemos llevar a cabo ha requerido de dos fuentes de datos. En primer lugar, utilizaremos datos que proceden del EUSTAT, Instituto Vasco de Estadística, (EUSTAT, 1986), y constituyen la base para la realización de la Encuesta continua de Población en Relación con la Actividad (PRA), publicada trimestralmente por el EUSTAT. Disponemos de 32 periodos trimestrales, comprendidos entre el primer trimestre de 2005 (2005-I) y el último trimestre de 2012 (2012-IV). La base de datos se basa en una muestra probabilística continua; es decir, un panel de viviendas que se va renovando continuamente. La muestra actual de la PRA tiene un tamaño aproximado de cinco mil viviendas por trimestre (un total aproximado de 13500 individuos) y una rotación de un octavo de un trimestre a otro. Es decir, el mismo individuo permanece en la muestra durante ocho periodos trimestrales (dos años), y después desaparece.

El conjunto de datos cuenta con un gran número de variables, que podemos clasificar en distintas categorías: características demográficas, familiares y sociales; apartado relativo a la formación; apartado relativo a la actividad laboral; apartado relativo a la actividad no laboral; y apartado relativo a la búsqueda de empleo y disponibilidad laboral. En total, disponemos de 33 variables a lo largo de 32 periodos trimestrales para un total de 13500 individuos por trimestre. En el caso que nos ocupa, utilizaremos una pequeña parte de la misma, pero resulta interesante por sí misma para poder realizar un análisis exhaustivo del mercado laboral vasco en distintas facetas (Oguiza et al., 2012). Hemos decidido trabajar con datos anuales para obtener una visión global de la evolución en la tasa de ocupación durante el periodo 2005-2012, por lo que hemos promediado los datos trimestrales. Así, hemos creado los datos de 8 periodos, de 2005 a 2012. Adicionalmente, creemos que el periodo es interesante por sí mismo, dado que incluye el fin de una etapa económica expansiva y el comienzo de una crisis económica, en 2008, que se prolonga hasta el final de nuestra muestra, 2012.

No obstante, dicha base de datos no recoge información sobre la nacionalidad o procedencia del individuo estudiado. Después de una consulta 
al EUSTAT, nos indicaron que no es posible facilitar dicho dato por una norma de confidencialidad que siguen. Por ello, hemos consultado otra fuente estadística, el Instituto Nacional de Estadística español, que publica trimestralmente la Encuesta de Población Activa. Dicha fuente de datos proporciona las tasas de ocupación por nivel de estudios y procedencia geográfica para hombres y mujeres. En lo que al presente trabajo se refiere, hemos calculado, por un lado, la tasa de ocupación de los hombres y mujeres inmigrantes que provienen de Europa, América, África y Asia, para poder compararlos con el mismo colectivo del País Vasco. Por otro lado, hemos obtenido la tasa de ocupación desagregada por nivel de estudios para los inmigrantes de Europa y América. Dado que la muestra se reducía considerablemente y creemos que no resultaba fiable, hemos descartado, en este caso, a los inmigrantes africanos y asiáticos.

Por último, a pesar de contar con datos anuales entre 2005-2012, hemos decidido enfocar el análisis a estudiar el efecto del sexo y de una educación superior en las tasas de ocupación en época de expansión económica con respecto a la época de recesión económica. Por ello, hemos dividido la muestra en dos periodos: 2005-2008, al que denominaremos periodo de Pre-Crisis y, 2009-2012, denominado periodo de crisis. El objetivo, así, consiste en estudiar cómo se han visto afectadas las tasas de ocupación de la población inmigrante (europeos, americanos, africanos y asiáticos) y la población autóctona del País Vasco, de un periodo de expansión económica (2005-2008), a un periodo de recesión (2009-2012), todo ello relacionándolo con el sexo y el nivel de estudios de los distintos individuos.

\section{ANÁLISIS EMPírico}

\section{Evolución de la población inmigrante en el País Vasco}

En primer lugar, creemos que resulta interesante conocer cuál ha sido la evolución de la población inmigrante mayor de 16 años en el País Vasco en el periodo de estudio, 2005-2012. Mostramos en la Figura 1, el número de inmigrantes venidos al País Vasco procedentes de Europa, América, África y Asia.

En lo que a los inmigrantes europeos se refiere, en el periodo 20052007, aumentan considerablemente, mientras que entre 2007-2009, cae ligeramente el número de los mismos pero, entre 2009-2010, se reducen de manera notoria. Como conclusión global, se observa que el número de inmigrantes europeos al País Vasco en el año 2012 es ligeramente superior al que se registraba en 2005. 
Figura 1: Evolución de la población inmigrante en el País Vasco durante el periodo 2005-2012

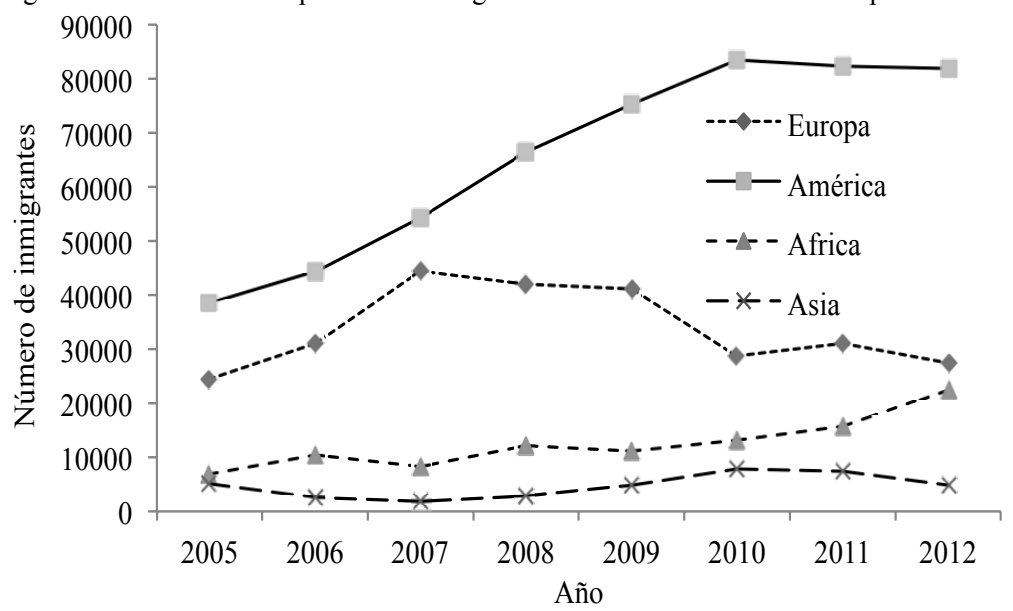

Fuente: elaboración propia a partir de la Encuesta de Población Activa (EPA), publicada por el Instituto Nacional de Estadística (INE).

Por otro lado, destacamos los inmigrantes americanos, en 2005, el censo de la población americana en el País Vasco era de 38594 personas y en 2012 de 81815 personas, por lo que en el periodo estudiado se duplican las personas procedentes de América. Entre 2005 y 2010 se observa un aumento constante y considerable de inmigrantes americanos, de manera que el flujo de población americana al País Vasco sigue aumentando en época de crisis, si bien entre 2010-2012, disminuye ligeramente. Entre los inmigrantes africanos no parece observarse ningún patrón decreciente en función de la crisis económica, podemos señalar que en 2005 y 2012 había 6895 y 22633 inmigrantes africanos, respectivamente, por lo que, de manera global, el número de inmigrantes africanos se triplica a lo largo del periodo. Por último, la inmigración asiática es baja y muy estable a lo largo de todo el periodo.

Como conclusión global observamos que el País Vasco recibe mayoritariamente población inmigrante americana, seguida de la europea y a cierta distancia están los africanos y asiáticos.

Adicionalmente, podemos completar la información de la Figura 1, basándonos en la estadística de movimientos migratorios publicada por el EUSTAT y Aierdi (2014), matizando en la procedencia de los distintos inmigrantes. En primer lugar, en el año 2005, la mitad de los extranjeros que venían al País Vasco, tenían nacionalidad de algún país americano, fundamentalmente Colombia y Ecuador; la cuarta parte correspondía a extranjeros europeos (en su mayoría portugueses) y cerca de la quinta parte 
provenía del continente africano (más de la mitad de ellos eran marroquíes), ocho por ciento restante eran de nacionalidad asiática, mayoritariamente china. Sin embargo, para el año 2010, la composición por áreas de nacionalidad de la inmigración extranjera en el País Vasco estaba experimentando un reajuste. Por un lado, la población magrebí estaba ganando terreno tanto en términos absolutos como relativos, lo contrario que ocurre con los europeos. Destacamos, entre los inmigrantes europeos, a los rumanos, recordando que Rumanía pasó a formar parte de la Unión Europea el 1 de enero de 2007. A partir de entonces, la población rumana ha pasado a ser la más importante entre la inmigración europea. Entre los inmigrantes americanos, los de Colombia y Ecuador ya están prácticamente instalados, mientras que el caso de Bolivia representa una irrupción inmediata que luego se ralentiza, y nacionalidades como Paraguay o Nicaragua experimentan un modelo similar al de la nacionalidad boliviana, con la variante de que estas dos últimas sufren una menor aceleración por causa del momento de llegada económicamente menos propicio. En la población asiática, conviene resaltar que, si bien históricamente ha sido la población china la nacionalidad más relevante desde un punto de vista absoluto de aportación de personas inmigrantes, en los último años comienza a despuntar la población de nacionalidad pakistaní (Aierdi, 2014).

\section{TASA DE OCUPACIÓN}

\section{Tasa de ocupación por procedencia geográfica y sexo}

En primer lugar, calculamos la tasa de ocupación de la población vasca durante el periodo 2005-2012. La tasa de ocupación la hemos calculado usando la metodología propuesta por el Instituto Vasco de Estadística y el Instituto Nacional de Estadística, como el número de ocupados dividido entre la población de 16 a 64 años (edad cumplida). Hemos decidido hacerlo así para que resulte comparable con la tasa de ocupación que calcula el Instituto Europeo de Estadística (EUROSTAT), el cual lo hace para la población de 15 a 64 años. Para poder realizar una comparación directa entre la fuente estadística europea, EUROSTAT, y las estadísticas obtenidas mediante el cálculo realizado en el trabajo, incluimos esta información en la Tabla 1, donde observamos las tasas de ocupación femenina y masculina para los años 2005 y 2012. En ella podemos apreciar que las tasas de ocupación calculadas en este estudio y las calculadas haciendo uso de la metodología del EUROSTAT son muy similares. 
Efectos de la crisis económica en el empleo de la población inmigrante en el País Vasco: un análisis... / V. NUÑEZ et al.

Tabla 1: Comparación de las tasas de ocupación por sexo. Años 2005 y 2012

\begin{tabular}{lrrrr}
\hline & \multicolumn{2}{c}{2005} & \multicolumn{2}{c}{2012} \\
\hline & Hombres & Mujeres & Hombres & Mujeres \\
\hline Cálculo propio & 74.7 & 53.0 & 66.3 & 57.3 \\
EUROSTAT & 76.4 & 54.6 & 66.9 & 58.1 \\
\hline
\end{tabular}

Fuente: cálculos propios a partir de datos de la Encuesta de Población en Relación con la Actividad (EPRA) publicada por Eurostat.

A continuación procedemos a analizar las tasas de ocupación diferenciando por sexo, en el País Vasco y en la población inmigrante de Europa, América, África y Asia, en el periodo de pre-crisis (2005-2008) y crisis (2009-2012). Los datos correspondientes se pueden hallar en la Tabla 2. En concreto, queremos estudiar si existen diferencias significativas en la tasa de ocupación masculina y femenina para poder analizar si el efecto de la crisis es distinto en ambos grupos.

Tabla 2: Tasa de ocupación por procedencia geográfica y sexo

\begin{tabular}{|c|c|c|c|c|c|c|c|c|c|c|}
\hline & \multicolumn{2}{|c|}{ País Vasco } & \multicolumn{2}{|c|}{ Europa } & \multicolumn{2}{|c|}{ América } & \multicolumn{2}{|c|}{ África } & \multicolumn{2}{|c|}{ Asia } \\
\hline & $\mathrm{H}$ & $\mathrm{M}$ & $\mathrm{H}$ & $\mathrm{M}$ & $\mathrm{H}$ & $\mathrm{M}$ & $\mathrm{H}$ & $\mathrm{M}$ & $\mathrm{H}$ & M \\
\hline Pre-Crisis & 74.9 & 55.4 & 73.0 & 55.2 & 62.5 & 62.9 & 81.3 & 31.2 & 68.8 & 49.0 \\
\hline Crisis & 69.2 & 57.3 & 67.8 & 53.6 & 56.9 & 61.6 & 43.3 & 11.7 & 77.5 & 77.5 \\
\hline
\end{tabular}

La tasa de ocupación masculina en el País Vasco era de 75 por ciento en el periodo antes de la crisis y pasa a ser algo más de 69 por ciento después de la crisis, acusando, por tanto, los hombres vascos el efecto de la recesión económica. No ocurre lo mismo entre las mujeres vascas, si bien antes de la crisis el porcentaje de ocupación femenina era de 55 por ciento (20 puntos inferior a la masculina), después de la crisis se registra una tasa de ocupación ligeramente superior, de algo más de 57 por ciento. Por tanto, se constata un comportamiento opuesto de las mujeres y hombres vascos ocupados frente a la crisis económica, siendo las primeras las que repuntan levemente su porcentaje de ocupación frente a la caída de la misma en el sector masculino. De hecho, esto es un reflejo de lo que lleva ocurriendo en la economía vasca en los últimos 20 años, la progresiva incorporación de la mujer al mundo laboral, así como su capacidad para resistir mejor los periodos de recesión (Oguiza et al., 2004).

A continuación, resulta relevante el análisis de la población inmigrante en el País Vasco, en el sentido de si ésta sigue unas pautas parecidas a la 
población autóctona, o si, por el contrario, muestra una evolución diferente. En lo que a la población inmigrante europea se refiere, la tasa de ocupación masculina es 18 puntos superior a la femenina en el periodo pre-crisis (73 y 55 por ciento, respectivamente), pasando esta diferencia a ser de 14 puntos en el periodo de recesión (casi 68 y 54 por ciento, respectivamente). A pesar de acusar un descenso en la tasa de ocupación, tanto para los hombres como para las mujeres, se puede observar que la población ocupada femenina europea resulta afectada en menor medida. En este sentido, podemos concluir que la población vasca y los inmigrantes europeos tienen un comportamiento similar en sus tasas de ocupación masculina y femenina, y en su comportamiento ante la crisis económica.

La población inmigrante americana destaca por el hecho de que el porcentaje de ocupación femenina y masculina en época de expansión es prácticamente el mismo (casi 63 por ciento las primeras y 62.5 por ciento los segundos) y, en época de recesión, esta diferencia aumenta, superando la tasa de ocupación femenina en casi cinco puntos a la masculina. Por tanto, resulta interesante destacar que las mujeres inmigrantes americanas tienen un porcentaje de ocupación mayor a los hombres, tanto antes como después de la crisis, poniendo de manifiesto la feminización de la ocupación procedente de América.

En el caso de la población inmigrante africana y asiática, los resultados se deben analizar con cierta cautela, dado que el tamaño muestral se reduce considerablemente y, pequeños cambios en la muestra tienen un gran efecto. Entre los inmigrantes africanos, cabe destacar que, a diferencia de los americanos, la tasa de ocupación masculina es considerablemente superior a la femenina, en el periodo de pre-crisis, 50 puntos superior la masculina a la femenina: un porcentaje de ocupados de más de 81 frente a 31 por ciento de ocupadas. No obstante, el hombre inmigrante africano sufre de manera considerable el efecto de la recesión, reduciéndose su tasa de ocupación en 38 puntos porcentuales en el periodo de crisis. La tasa de ocupación de las mujeres inmigrantes africanas no se reduce en la misma medida (casi 20 puntos porcentuales), si bien su tasa de ocupación de partida era considerablemente menor. Así, en época de crisis los hombres africanos registran una tasa de ocupación de algo más de 43 por ciento, frente a casi 12 por ciento femenina. En el caso de la población asiática, la diferencia en el porcentaje de ocupados entre hombres y mujeres no es tan acusada como en el caso de África, en el periodo pre-crisis, los primeros registraban una tasa de ocupación de casi 70 por ciento y, las segundas, de 49 por ciento (una diferencia de casi 20 puntos porcentuales). Este es el único colectivo que 
en conjunto mejora su ocupación en el periodo de recesión equilibrándose, además, en el periodo de crisis la ocupación masculina y femenina.

Dado que hemos analizado la tasa de ocupación masculina y femenina en periodo de Pre-Crisis y de Crisis, entre la población autóctona del País Vasco y la población inmigrante europea, americana, africana y asiática, para poder completar el análisis, hemos considerado que sería interesante observar el número de inmigrantes que viene al País Vasco, de las distintas procedencias estudiadas, con el objeto de conocer en valor absoluto, el movimiento migratorio de estas poblaciones. La Tabla 3 muestra la evolución del número de inmigrantes por sexo.

Tabla 3: Número de inmigrantes por procedencia geográfica y sexo

\begin{tabular}{|c|c|c|c|c|c|c|c|c|}
\hline & \multicolumn{2}{|c|}{ Europa } & \multicolumn{2}{|c|}{ América } & \multicolumn{2}{|c|}{ África } & \multicolumn{2}{|c|}{ Asia } \\
\hline & $\mathrm{H}$ & M & $\mathrm{H}$ & $\mathrm{M}$ & $\mathrm{H}$ & $\mathrm{M}$ & $\mathrm{H}$ & $\mathrm{M}$ \\
\hline Pre-crisis & 17410 & 18058 & 19194 & 31674 & 6535 & 2886 & 1446 & 1645 \\
\hline Crisis & 16247 & 15826 & 32308 & 48377 & 9369 & 6294 & 4410 & 1884 \\
\hline
\end{tabular}

Los aspectos que consideramos destacables de esta información son:

- En primer lugar, considerando entre toda la población inmigrante, solamente en el caso de los europeos, se reduce el censo de población tanto masculina como femenina del periodo de expansión (2005-2008) al periodo de crisis (2009-2012). En el resto, tanto entre los americanos, africanos, como asiáticos, a pesar de la crisis económica que sufre el País Vasco a partir de 2008, el censo de estas poblaciones tanto en los hombres como en las mujeres, aumenta.

- Respecto a los distintos orígenes de inmigración considerados, destaca la inmigración americana al País Vasco por encima de todas las demás. Además, dentro de ésta, se constata la feminización de la población inmigrante, casi duplicando a la masculina en el periodo de pre-crisis. Este hecho junto con lo observado en el estudio de la población ocupada, donde constatábamos que la tasa de ocupación femenina mayor correspondía a las mujeres americanas y eran, precisamente éstas, las que mejor resistían el efecto de la crisis, enfatiza la importancia de la inmigración de la mujer americana al País Vasco, así como su capacidad de adaptación en el periodo de recesión económica. Este hecho se apoya, además, en la constatación de que son éstas las que conforman la mayoría de la población inmigrante femenina. 
- La última característica reseñable es el censo de población inmigrante asiática, ya que éste es considerablemente bajo, tanto para hombres como para mujeres. En este caso, cabe mencionar que el número de hombres inmigrantes asiáticos se triplica del periodo de pre-crisis al periodo de crisis, y el de las mujeres aumenta ligeramente, no viéndose influenciados por la recesión económica.

Como conclusión, observamos que la mayor aportación de personas inmigrantes lo constituye la mujer americana, muy por encima del resto de procedencias geográficas, aumentando incluso en época de recesión. Además, es la mujer americana, junto a la asiática, la que registra una mayor tasa de ocupación y es la que mejor se comporta frente a la crisis económica. El censo de población inmigrante europea se reduce en el periodo de recesión, siendo en este caso, también la mujer, la que acusa en menor medida el periodo de recesión económica, en términos de ocupación. La población procedente de África, es en mayor medida masculina, con una tasa de ocupación muy superior a la femenina, si bien, en este caso, se ven muy afectados con la llegada de la crisis económica.

A la vista de los resultados obtenidos, resulta interesante analizar en qué sector de actividad económica trabaja cada grupo, con objeto de entender mejor la evolución de la tasa de ocupación de los hombres y mujeres en los periodos de pre-crisis y crisis económica.

\section{Tasa de ocupación por procedencia geográfica, sexo y sector de actividad}

Analizamos, a continuación, la tasa de ocupación masculina y femenina, en las distintas áreas geográficas estudiadas diferenciando por los cuatro grandes sectores de actividad económica: agricultura, industria, construcción y servicios. Las Tablas 4, 5, 6 y 7 presentan dichas tasas.

El análisis por sector de actividad económica constata que, de manera global, hombres y mujeres trabajan en distintos sectores, tanto los residentes en el País Vasco, como los inmigrantes, con ciertas matizaciones.

Por lo que respecta a los trabajadores vascos, la ocupación masculina está dividida mayoritariamente entre industria ( 26 por ciento antes de la crisis, cayendo dos puntos porcentuales en la crisis) y servicios (con un 38 por ciento de ocupación, también descendiendo más de dos puntos en recesión). Las mujeres vascas se encuentran trabajando primordialmente en el sector de servicios, 45 por ciento en pre-crisis, destacando que la ocupación femenina aumenta casi cinco puntos en el periodo de crisis. 
Efectos de la crisis económica en el empleo de la población inmigrante en el País Vasco: un análisis... / V. NUÑEZ et al.

Tabla 4: Tasa de ocupación por procedencia geográfica, sexo y sector de actividad Agricultura

\begin{tabular}{lrrrrrrrrrr}
\hline & \multicolumn{2}{c}{ País Vasco } & \multicolumn{2}{c}{ Europa } & \multicolumn{2}{c}{ América } & \multicolumn{2}{c}{ África } & \multicolumn{2}{c}{ Asia } \\
\hline & H & M & H & M & H & M & H & M & H & M \\
\hline Pre-crisis & 1.4 & 0.5 & 2.3 & 0.0 & 3.1 & 0.2 & 5.9 & 2.5 & 0.0 & 0.0 \\
Crisis & 1.0 & 0.4 & 0.7 & 0.0 & 0.8 & 0.0 & 0.6 & 0.7 & 0.0 & 0.0 \\
\hline
\end{tabular}

Nota: H: Hombre, M: Mujer. Pre-crisis años 2005-2008 y crisis años 2009-2012.

Fuente: elaboración propia a partir de la Encuesta de Población Activa (EPA), publicada por el Instituto Nacional de Estadística (INE).

Tabla 5: Tasa de ocupación por procedencia geográfica, sexo y sector de actividad Industria

\begin{tabular}{lrrrrrrrrrr}
\hline & \multicolumn{1}{c}{ País Vasco } & \multicolumn{2}{c}{ Europa } & \multicolumn{3}{c}{ América } & \multicolumn{2}{c}{ África } & \multicolumn{2}{c}{ Asia } \\
\hline & H & M & H & M & H & M & H & M & H & M \\
\hline Pre-crisis & 26.4 & 8.4 & 13.6 & 0.6 & 10.1 & 1.3 & 33.1 & 0.0 & 1.9 & 6.9 \\
Crisis & 24.4 & 6.1 & 14.5 & 1.9 & 5.0 & 3.2 & 14.2 & 0.0 & 0.0 & 0.0 \\
\hline
\end{tabular}

Nota: H: Hombre, M: Mujer. Pre-crisis años 2005-2008 y crisis años 2009-2012.

Fuente: elaboración propia a partir de la Encuesta de Población Activa (EPA), publicada por el Instituto Nacional de Estadística (INE).

Tabla 6: Tasa de ocupación por procedencia geográfica, sexo y sector de actividad Construcción

\begin{tabular}{|c|c|c|c|c|c|c|c|c|c|c|}
\hline & \multicolumn{2}{|c|}{ País Vasco } & \multicolumn{2}{|c|}{ Europa } & \multicolumn{2}{|c|}{ América } & \multicolumn{2}{|c|}{ África } & \multicolumn{2}{|c|}{ Asia } \\
\hline & $\mathrm{H}$ & M & $\mathrm{H}$ & M & $\mathrm{H}$ & M & $\mathrm{H}$ & M & $\mathrm{H}$ & M \\
\hline Pre-crisis & 9.1 & 1.5 & 33.5 & 0.0 & 18.7 & 0.1 & 16.0 & 0.0 & 11.1 & 0.0 \\
\hline Crisis & 8.1 & 0.9 & 21.9 & 0.0 & 14.5 & 0.0 & 8.2 & 0.8 & 6.0 & 0.0 \\
\hline
\end{tabular}

Tabla 7: Tasa de ocupación por procedencia geográfica, sexo y sector de actividad Servicios

\begin{tabular}{lrrrrrrrrrrr}
\hline & \multicolumn{1}{c}{ País Vasco } & \multicolumn{2}{c}{ Europa } & \multicolumn{2}{c}{ América } & \multicolumn{2}{c}{ África } & \multicolumn{3}{c}{ Asia } \\
\hline & H & M & H & M & H & M & H & M & H & M \\
\hline Pre-crisis & 38.1 & 45.1 & 23.6 & 54.6 & 30.6 & 61.3 & 26.3 & 28.7 & 55.8 & 42.0 \\
Crisis & 35.7 & 49.9 & 30.8 & 51.7 & 36.5 & 58.5 & 20.2 & 10.2 & 71.5 & 77.5 \\
\hline
\end{tabular}

Nota: H: Hombre, M: Mujer. Pre-crisis años 2005-2008 y crisis años 2009-2012.

Fuente: elaboración propia a partir de la Encuesta de Población Activa (EPA), publicada por el Instituto Nacional de Estadística (INE). 
Observando a la población inmigrante, en la ocupación de los hombres europeos predominan los sectores de la construcción y los servicios con cierta presencia en la industria. Antes de la recesión económica, 33.5 y casi 24 por ciento de los hombres inmigrantes europeos trabajaban en los sectores de construcción y servicios, respectivamente. Durante el periodo de crisis, los ocupados en la construcción caen casi 12 puntos porcentuales, mientras que en el sector servicios suben siete puntos. Las mujeres de este colectivo se concentran en el sector servicios con 54.6 por ciento de ocupación, cayendo tres por ciento y su presencia en el resto de sectores es prácticamente testimonial.

Los hombres inmigrantes americanos siguen una pauta similar a los europeos, no obstante, la tasa de ocupación antes de la crisis en el sector de la construcción era menor, a saber, 19 por ciento y ésta disminuye cuatro puntos en recesión. En el sector servicios aumenta seis puntos. Las mujeres de este grupo, siguen el mismo patrón que las europeas pero con una tasa de ocupación mayor, más de 61 por ciento de ocupación femenina en los servicios cayendo tres por ciento con la crisis.

En relación a los hombres africanos, observamos que la ocupación está más dividida entre los distintos sectores, pero entre ambos periodos estudiados, podríamos decir que la ocupación se desploma en todos ellos. Las tasas de ocupación en los periodos pre-crisis y crisis son, respectivamente, en agricultura casi seis por ciento, pasa a uno por ciento; construcción de 16 a ocho por ciento; industria de 33 a 14 por ciento y, por último en servicios de 26 a 20 por ciento. En el caso de las mujeres africanas, la ocupación se encuentra básicamente en el sector de servicios, con una tasa de empleo de casi 29 por ciento antes de la crisis, reduciéndose ésta de manera notoria a diez por ciento en época de recesión.

Por último, presentamos los resultados para los ciudadanos procedentes de Asia por coherencia con el trabajo, pero en este caso al desagregar por sexo y sector de actividad, la muestra se reduce notablemente y debemos tomar los resultados con cautela. Los hombres asiáticos se ocupan principalmente en el sector servicios y en menor medida en la construcción. A raíz de la crisis aumenta su concentración en los servicios. Las mujeres asiáticas se concentran prácticamente en los servicios, con un aumento de su ocupación de 35 puntos porcentuales con la crisis.

Este análisis de la tasa de ocupación por sector de actividad económica nos permite dar una explicación al hecho de que, globalmente, las mujeres ocupadas son las que han resistido el efecto de la crisis económica frente a los hombres que han resultado más afectados, tal y como hemos visto. Se 
observa, que, en general, hombres y mujeres se encuentran trabajando en distintos sectores de actividad económica y esta crisis ha recaído en primer lugar sobre la construcción (sector de ocupación primordialmente masculina) y, en segundo lugar, en la industria (también con mayoritaria presencia de hombres). El sector de servicios ha sido la actividad que mejor ha resistido el embate de la recesión. Antes de la crisis económica, la ocupación femenina, tanto de la población autóctona como de las inmigrantes, se concentraba en los servicios, hecho que explica la mejor situación relativa de la mujer frente al hombre ocupado en el periodo de crisis. De hecho, la actividad de los servicios, pasa a ser en la crisis económica el principal sector de ocupación masculina.

\section{Tasas de ocupación por procedencia geográfica y nivel de estudios}

Una vez estudiada la tasa de ocupación por sexo, procedencia geográfica y sector de actividad, otra característica relevante de los individuos ocupados es el nivel de educación de los mismos. Consideramos tres niveles de formación: estudios primarios, secundarios y universitarios. Ésta es la clasificación que hacen tanto el Instituto Vasco de Estadística, como el Instituto Nacional de Estadística. A continuación, queremos estudiar si un nivel de educación superior contribuye a una mejora en la tasa de ocupación, tanto de la población autóctona del País Vasco, como de los individuos procedentes de las distintas áreas geográficas consideradas.

Dado que el censo de población para los inmigrantes africanos y asiáticos diferenciando por nivel de estudios se reduce considerablemente y consideramos que el análisis podría verse afectado por este hecho (tal y como explicaremos más adelante y mostramos), en este caso, hemos decidido comparar a los individuos del País Vasco con la población procedente de Europa y América.

En la Tabla 8, se muestra la evolución en la tasa de ocupación de los individuos del País Vasco y de la población procedente de Europa y América, desagregando por el nivel de formación de los individuos.

En primer lugar, hemos considerado interesante estudiar, dentro de cada grupo de procedencia geográfica, si distintos niveles de estudio repercuten de diferente manera en las tasas de ocupación de los mismos. En este sentido, se observa que entre la población vasca, son los individuos con un nivel de formación mayor los que mejor resisten la crisis en términos de ocupación, manteniéndose su tasa de ocupación en época de crisis. Entre la población procedente de Europa, los universitarios acusan más el efecto de la recesión (cayendo 8.6 puntos porcentuales) y, son los individuos con 
estudios secundarios los que mejor se comportan, aumentando incluso más de tres por ciento su ocupación. Por último, destacamos que para la población originaria de América, podemos observar que tanto la población con estudios secundarios como universitarios, no parecen acusar de manera considerable el efecto de la crisis.

Tabla 8: Tasa de ocupación por procedencia geográfica y nivel de estudios

\begin{tabular}{lrrrrrrrrr}
\hline & \multicolumn{3}{c}{ País Vasco } & \multicolumn{3}{c}{ Europa } & \multicolumn{3}{c}{ América } \\
\hline & Prima & Secun & Univer & Prima & Secun & Univer & Prima & Secun & Univer \\
\hline Pre-crisis & 45.4 & 67.6 & 76.3 & 50.2 & 62.1 & 75.0 & 60.5 & 61.5 & 69.4 \\
Crisis & 40.4 & 63.0 & 76.9 & 33.9 & 65.4 & 66.4 & 48.5 & 60.6 & 66.5 \\
\hline
\end{tabular}

Nota: Prima: nivel de estudios primarios; Secun: nivel de estudios secundarios; Univer: nivel de estudios universitarios.

Pre-crisis años 2005-2008 y Crisis años 2009-2012.

Fuente: elaboración propia a partir de la Encuesta de Población Activa (EPA), publicada por el Instituto

Nacional de Estadística (INE).

En segundo lugar, otro rasgo fundamental reside en comparar los distintos niveles de formación, por procedencia geográfica. Se observa que, entre la población autóctona y los inmigrantes europeos, en el periodo de pre-crisis, la menor tasa de ocupación la arrojan los individuos con un menor nivel de estudios, primarios ( 45 y 50 por ciento, respectivamente), después el nivel de estudios secundarios (casi 68 y 62 por ciento, respectivamente) y, son los universitarios los que cuentan con un porcentaje de ocupados mayor (76 y 75 por ciento, respectivamente). Si consideramos la tasa de ocupación de la población inmigrante americana, los individuos universitarios, también en este caso, disfrutan del mayor porcentaje de ocupación (60.5 por ciento los individuos con estudios primarios, 61.5 por ciento los secundarios y 69 por ciento los universitarios). La pregunta que consideramos interesante es, si un mayor nivel de formación resulta positivo en época de crisis, esto es, si una inversión en capital humano supone una mejora positiva en la tasa de ocupación del mismo. En este sentido, observamos que la población con estudios primarios es, definitivamente, la que acusa el efecto de la crisis en mayor medida, sea cual sea el origen del individuo. Así, del periodo de pre-crisis al periodo de crisis, la tasa de ocupación de los mismos se reduce en cinco puntos porcentuales para los individuos vascos, más de 16 puntos porcentuales para los europeos y 12 puntos para los americanos.

Por tanto, en resumen, los individuos que mejor resisten los efectos de la crisis en términos de ocupación son los vascos universitarios, los europeos con estudios secundarios y los americanos con estudios secundarios y universitarios. Se constata, por tanto, que una mayor formación del indi- 
viduo resulta beneficioso en términos de ocupación, en época de crisis, por lo que una mayor inversión en capital humano genera una posición relativa favorable en el mercado laboral.

Resulta interesante, tal y como hemos hecho al explicar las tasas de ocupación por sexo, mostrar los censos de población por nivel de estudios y procedencia geográfica, para poder tener una visión del número total de población que tenemos en cada caso. Dichos datos se muestran en la Tabla 9 , incluimos también a la población inmigrante africana y asiática con el objeto de ver que el censo de población en estos casos se reduce considerablemente.

Tabla 9: Número de inmigrantes por procedencia geográfica y nivel de estudios

\begin{tabular}{|c|c|c|c|c|c|c|c|c|c|c|c|c|}
\hline & & Europa & & & América & & & África & & & Asia & \\
\hline & Prima & Secun & Univer & Prima & Secun & Univer & Prima & Secun & Univer & Prima & Secun & Univer \\
\hline Pre-crisis & 6328 & 18398 & 10743 & 9058 & 31545 & 10266 & 4608 & 3608 & 1205 & 882 & 963 & 1246 \\
\hline Crisis & 5706 & 17191 & 9176 & 13360 & 53296 & 14029 & 6518 & 7979 & 1166 & 2626 & 3105 & 563 \\
\hline
\end{tabular}

Destacamos los siguientes aspectos:

- Tal y como hemos señalado al explicar la inmigración por sexo, el censo de población europea se reduce en todos los niveles de estudio en época de crisis.

- El hecho reseñable en el estudio reside en la inmigración americana con estudios secundarios, arrojando el censo de población mayor entre todos los inmigrantes, aumentando, además, considerablemente en época de crisis. Recordemos, además, que es precisamente la mujer americana con estudios secundarios la que arroja una mayor tasa de ocupación y presenta un comportamiento más favorable frente a la crisis económica.

- Por último comentar que tanto la población inmigrante africana como asiática con estudios primarios y secundarios aumenta su censo de población en la época de recesión, si bien los universitarios en ambos casos, se reducen. Estos resultados los observamos, no obstante, con la debida cautela, dado su número reducido. 


\section{CONCLUSIONES FinALES}

En este artículo se ha realizado un estudio sobre las tasas de ocupación de la población inmigrante en el País Vasco en el periodo 2005-2012, un periodo que se divide en dos etapas, una de expansión económica (20052008) y otra posterior de recesión económica (2009-2012). El objetivo ha consistido en observar el comportamiento de la ocupación de la población inmigrante durante la crisis y cuantificar las diferencias sobre la misma según sexo, procedencia y nivel de estudios, y sus diferencias con respecto a la población autóctona. Independientemente de su sexo, origen o cualificación, al final de la crisis la tasa de ocupación de los inmigrantes es menor que la de la población vasca, con la única excepción de las mujeres de procedencia americana y asiática. Además, el análisis realizado revela que el impacto de la crisis sobre la ocupación de los inmigrantes en el País Vasco varía considerablemente entre los grupos según sexo, origen, nivel de estudios y sector de actividad.

Se puede observar que la tasa de ocupación masculina es siempre mayor que la femenina para todos los trabajadores, autóctonos y foráneos, con la excepción de los de origen americano; si bien esta diferencia disminuye a raíz de la crisis, aumentando la ventaja en el caso de las mujeres americanas. Esta disminución puede ser debida al efecto trabajador añadido, a diferencias en los niveles educativos o a la segregación ocupacional de las mujeres, concentradas en el sector servicios menos expuesto a la crisis económica.

A la vista del análisis de la ocupación por sectores económicos parece que la segregación sectorial es la razón que ha podido tener un mayor peso a la hora de explicar la caída en la brecha de género en la ocupación. La ocupación de las mujeres inmigrantes fuera del sector servicios es casi nula independientemente de su procedencia, circunstancia que no ha cambiado con la recesión. Para los hombres existen importantes diferencias según origen y sector económico pero, en general, el sector servicios suele tener los menores descensos en ocupación e incluso alzas en la misma, a pesar de la crisis. Si analizamos la ocupación de los inmigrantes hombres según su origen podemos observar que los africanos son los peor parados con la recesión, con una gran caída de la ocupación en todos los sectores económi$\cos$. En el otro extremo se encuentran los trabajadores asiáticos que incluso aumentan su ocupación, probablemente debido a su concentración en el sector servicios, reforzando la idea de que la segregación ocupacional puede ser la mejor explicación a las diferencias tras la crisis. Por último, tra- 
bajadores con origen europeo y americano se encuentran en una situación intermedia, con caídas en la ocupación similares a las de los trabajadores vascos y una presencia más repartida en todos los sectores económicos.

La evidencia empírica sugiere una relación positiva entre nivel de estudios y ocupación de los inmigrantes (véase, por ejemplo, Valero et al., 2014, para un análisis en el mercado laboral español). La crisis económica ha tenido un fuerte impacto en los trabajadores con estudios primarios, tanto inmigrantes como locales, con importantes caídas en las tasas de ocupación. El efecto de tener estudios universitarios es menos claro y, si bien para los trabajadores vascos resulta un escudo ante el efecto de la crisis, los trabajadores inmigrantes con estudios universitarios de origen americano y europeo, los analizados, presentan caídas en la ocupación mayores que los que tienen estudios secundarios, perdiendo presencia en los segmentos más cualificados de empleo.

En resumen podríamos señalar que los grandes perdedores con la crisis han sido los trabajadores africanos. Este resultado está en línea con lo que sugiere la teoría de la asimilación segmentada (Portes y Zhou, 1993) según la cual los inmigrantes de culturas más distantes a la del país de acogida pueden encontrarse con dificultades a la hora de integrarse en el mismo, en este caso dando lugar a un peor encaje en el mercado laboral. En diversos estudios a nivel europeo (véase Kahanec y Guzi, 2017; Gorodzeisky y Semyonov, 2017, entre los más recientes) se observa un resultado similar, con inmigrantes de origen europeo y asiáticos sorteando la crisis mejor que los de origen africano o americano. En este sentido el resultado de este estudio presenta una diferencia significativa con respecto a la inmigración de origen americano, sobre todo en el caso de las mujeres, que presentan la tasa de ocupación más alta con respecto a todos los demás grupos, incluidas las trabajadoras vascas y exceptuando a las mujeres asiáticas tras la crisis. Esta situación también se vería explicada por la teoría de la asimilación segmentada, ya que a diferencia de lo que ocurre en otros países europeos, los inmigrantes americanos se encuentran más cercanos a la cultura local.

Ahora bien, a pesar de esta mejor resistencia a la crisis de las inmigrantes americanas, efecto que se podría extender a las mujeres en general, éstas se encuentran confinadas en unas pocas ocupaciones, poco cualificadas, de empleo precario, salarios bajos y, en general, malas condiciones laborales. Sería necesario establecer políticas y acciones que permitan una mayor integración de las mujeres en distintas ocupaciones en distintos sectores económicos. Siendo las mujeres inmigrantes uno de los grupos más vulnerables tendrían que ser especialmente tenidas en cuenta. 
Otro grupo que necesitaría una especial atención son los inmigrantes africanos, el colectivo más vulnerable en el mercado laboral vasco. Los hombres son los que mayor caída en la ocupación han tenido a lo largo de la crisis y las mujeres africanas tienen un nivel de ocupación casi nulo, presentando los mayores problemas de asimilación al mercado laboral. Esta situación no es única del mercado vasco sino que se observa en otros países de la Unión Europea (véase, por ejemplo, Dumont e Isoppo, 2005). Así, las mujeres africanas presentan una doble desventaja, al proceder en muchos casos de países donde se suele restringir el papel de la mujer en el ámbito laboral y encontrarse con una sociedad cada vez más reacia a su asimilación. Para este colectivo no son necesarias únicamente políticas laborales, sino que habría que realizar acciones que permitan una mejor integración en la sociedad vasca.

\section{Agradecimientos}

Los autores agradecen al Instituto Vasco de Estadística (EUSTAT) por facilitar los datos. Este trabajo ha sido financiado por Ministerio de Economía y Competitividad, Agencia Estatal de Investigación (AEI), Fondo Europeo de Desarrollo Regional (FEDER), the Department of Education of the Basque Government (UPV/EHU Econometrics Research Group), and Universidad del País Vasco UPV/EHU under research grants MTM2013-40941-P (AEI/FEDER, UE), MTM2016-74931-P (AEI/FEDER, UE), ECO201676884-P (AEI/FEDER, UE), IT-642-13 and UFI11/03.

\section{REFERENCIAS BIBLIOGRÁFICAS}

Aierdi, X., 2014, Atzerriko biztanleak. Población extranjera. EUSTAT. VitoriaGasteiz.

Chaloff, J., Dumont, J. C. y Liebig, T., 2012, "The impact of the economic crisis on migration and labour market outcomes of immigrants in OECD countries", en CESifo DICE Report, vol. 10, núm. 1.

Cho, Y. y Newhouse, D., 2013, "How did the great recession affect different types of workers? Evidence from 17 middle-income countries", en World Development, vol. 41.

Dumont, J. C. e Isopppo, M., 2005, "The participation of immigrant women in the labour market: A double handicap despite the progress made", en Migrant Women and the Labour Market: Diversity and Challenges. OECD and European Commission. Brussels. 
EUSTAT, 1986, Encuesta Continua de la Población en Relación con la Actividad, Vitoria-Gasteiz: Eusko Jaurlaritza/Gobierno Vasco.

Eydoux, A., Math, A. y Périvier, H., 2014, "European labour markets in times of crisis: a gender perspective", en Revue de l'OFCE, vol. 133, núm. 2.

Farris, S. R., 2015, "Migrants' regular army of labour: gender dimensions of the impact of the global economic crisis on migrant labor in Western Europe", en The Sociological Review, vol. 63, núm 1.

Gorodzeisky, A. y Semyonov, M., 2017, “Labor force participation, unemployment and occupational attainment among immigrants in West European countries", en PloS one, vol. 12, núm. 5.

Ikuspegi, 2015, El impacto económico y demográfico de la inmigración extranjera en el País Vasco, Vitoria-Gasteiz: Eusko Jaurlaritza/Gobierno Vasco.

Kahanec, M. y Guzi, M., 2017, "How immigrants helped EU labor markets to adjust during the Great Recession", en International Journal of Manpower, vol. 38 , núm 7 .

Oguiza, A., Gallastegui, I. y Núñez-Antón, V., 2004, “La población ocupada en la CAPV (1993-1999). Género y formación como características relevantes", en Estadística Española, vol. 46, núm. 156.

Oguiza, A., Gallastegui, I. y Núñez-Antón, V., 2012, “Analysis of pseudo-panel data with dependent samples”, en Journal of Applied Statistics, vol. 39, núm 9.

Peña-Boquete, Y., 2014, "Have the economic crises reduced the gender gap on the Spanish labour market?", en Revue de l'OFCE, núm. 2.

Portes, A. y Zhou, M., 1993, “The new second generation: Segmented assimilation and its variants", en The annals of the American Academy of Political and Social Science, vol. 530, núm. 1.

Psacharopoulos, G. y Patrinos, H., 2002, Returns to investment in education: a further update World Bank Policy Research, Working Paper 2881. World Bank: Washington.

Sabarwal, S., Sinha, N. y Buvinić, M., 2011, "How do women weather economic shocks?: what we know", en World Bank, Poverty Reduction and Economic Management Network.

Valero-Matas, J. A., Romay-Coca, J. y Miranda, S., 2014, “Análisis de la inmigración en España y la crisis económica", en Papeles de Población, vol. 20, núm 80.

\section{RESUMEN CURRICULAR DE LOS AUTORES}

Vicente Núñez Antón

Profesor Catedrático de Estadística en el Departamento de Econometría y Estadística (Economía Aplicada III) de la Universidad del País Vasco UPV/EHU. Doctor en Estadística por la Universidad de Iowa, Estados Unidos. Imparte docencia sobre Inferencia Estadística y Modelos Lineales. 
Sus áreas de investigación incluyen el análisis de datos longitudinales, estimación no paramétrica y semiparamétrica, contrastes de bondad de ajuste, análisis de supervivencia y estudios de calidad de vida relacionados con la salud, en las que ha publicado numerosos artículos y varios libros.

Dirección electrónica: vicente.nunezanton@ehu.eus

\section{Ainhoa Oguiza Tovar}

Profesora Titular y Doctora por la Universidad del País Vasco/Euskal Herriko Unibertsitatea (UPV/EHU). Imparte docencia sobre Econometría y Estadística Descriptiva y Teórica. Colaboradora en el grupo de investigación consolidado "Econometrics Research Group", perteneciente a la UPV/EHU. Colaboradora del proyecto de investigación "Análisis de Datos longitudinales", perteneciente al Ministerio de Economía y Competitividad de España, dando lugar a varias publicaciones científicas.

Dirección electrónica: ainhoa.oguiza@ehu.eus

\section{Jorge Virto Moreno}

Profesor Titular de Escuela Universitaria en el Departamento de Econometría y Estadística (Economía Aplicada III) de la Universidad del País Vasco UPV/EHU, donde ejerce tareas docentes en Econometría y Estadística y Análisis de Datos. Sus principales temas de investigación y publicaciones en revistas incluyen contribuciones al análisis de la coyuntura económica, al estudio sobre la eficacia de distintos tratamientos en pacientes trasplantados renales, así como a la medición del valor del océano dentro del ámbito de la economía de los recursos naturales. Colabora además como investigador por la UPV/EHU en el consorcio europeo Marnet. Es también autor de varios libros sobre la práctica de la Estadística.

Dirección electrónica: jorge.virto@ehu.eus

Artículo recibido el 24 de noviembre de 2015 y aprobado el 16 de julio de 2017. 